\title{
$\mathrm{M}|\mathrm{R}| \mathrm{S}$ Internet Journal Nitride Semiconductor Research
}

\section{Indium incorporation and surface segregation during InGaN growth by molecular beam epitaxy: experiment and theory}

\author{
Huajie Chen ${ }^{1}$, R. M. Feenstra ${ }^{1}$, J. Northrup ${ }^{2}$, Jörg Neugebauer ${ }^{3}$ and D. W. Greve ${ }^{4}$ \\ ${ }^{1}$ Department of Physics, Carnegie Mellon University, \\ ${ }^{2}$ Xerox Palo Alto Research Center, \\ ${ }^{3}$ Fritz-Haber-Institut der MPG, \\ ${ }^{4}$ Department of Electrical and Computer Engineering, Carnegie Mellon University,
}

(Received Tuesday, June 5, 2001; accepted Tuesday, June 26, 2001)

InGaN alloys with (0001) or $(000 \overline{1})$ polarities are grown by plasma-assisted molecular beam epitaxy. Scanning tunneling microscopy images, interpreted using first-principles theoretical calculations, show that there is strong indium surface segregation on InGaN for both (0001) and (0001) polarities. Evidence for the existence and stability of a structure containing two adlayers of indium on the Inrich $\mathrm{InGaN}(0001)$ surface is presented. The dependence on growth temperature and group III/V ratio of indium incorporation in InGaN is reported, and a model based on indium surface segregation is proposed to explain the observations.

\section{Introduction}

Ternary $\operatorname{In}_{\mathrm{x}} \mathrm{Ga}_{1-\mathrm{x}} \mathrm{N}$ alloys are used as the active layer in GaN-based light emitting diodes (LEDs) and lasers [1]. It is thus important to understand and control the growth of InGaN. In our earlier work [2] we studied the dependence of In incorporation on growth parameters for InGaN with $(000 \overline{1})$ polarity. A qualitative model for the indium incorporation was proposed, based on observed strong surface segregation of the indium. In this paper, results for the dependence of indium incorporation on growth parameters are given for InGaN with (0001) polarity. It is found that the incorporation decreaseswhen the growth temperature is increased, it decreases when the group III/group $\mathrm{V}$ flux ratio is increased under metal rich conditions, and it increases as a function of this flux ratio under nitrogen rich conditions. A quantitative model is proposed to explain this dependence.

The basic InGaN surface structures discussed here are illustrated in Figure 1. Figure 1(a) shows the previously determined structure for the $\operatorname{InGaN}(000 \overline{1})$ surface, consisting of a monolayer (ML) of indium bonded to a $\mathrm{GaN}$ bilayer [2]. For less In-rich conditions this monolayer contains a mixture of indium and gallium. For the InGaN(0001) surface, prior studies indicate that the surface most commonly consists of two monolayers of metal atoms, with the top layer being entirely indium atoms and the second layer containing a mixture of indium and gallium atoms [3] [4]. This structure is illustrated in Figure 1(b).

\section{Experiment}

The studies described here were performed in a combined molecular beam epitaxy (MBE)/surface analysis system, as previously described [2] [4]. GaN(0001) was grown on $\mathrm{Si}$-face $6 \mathrm{H}-\mathrm{SiC}(0001)$ substrates, with the polish damage removed by $\mathrm{H}$-etching [5]. After the $\mathrm{H}$ etching, the substrate was introduced into the growth chamber and outgassed up to the temperature of $800^{\circ} \mathrm{C}$. A few monolayers of $\mathrm{Si}$ were deposited onto the surface and the substrate was then annealed to about $1000^{\circ} \mathrm{C}$ until a $\sqrt{3} \times \sqrt{3}$ reconstruction was obtained [6]. GaN was directly grown on this surface at growth temperature of $670^{\circ} \mathrm{C}$. Following the $\mathrm{GaN}$ growth with typical thickness of $200 \mathrm{~nm}$, the substrate temperature is lowered to $580-620^{\circ} \mathrm{C}$ for the InGaN deposition. $\mathrm{GaN}(000 \overline{1})$ was grown at $720^{\circ} \mathrm{C}$, on sapphire substrates, with pre-growth nitridation of the substrate performed at $1050^{\circ} \mathrm{C}$ and using a low-temperature $\mathrm{GaN}$ buffer layer grown at $550^{\circ} \mathrm{C}$. Typical growth rates for the $\mathrm{GaN}$ and InGaN are $200 \mathrm{~nm} / \mathrm{h}$. Gallium and indium flux rates were calibrated with an in situcrystal thickness monitor. The substrate temperature was measured by an optical pyrometer with emissivity set to be 0.7 . It should be noted that In and Ga coat the pyrometer window during growth, which affects the pyrometer reading gradually. 
Corrections for this effect were made to the temperatures used for $(000 \overline{1})$ polarity, but not for (0001) polarity. Also, the work on (0001) polarity was done one year after the work for $(000 \overline{1})$ polarity. Thus, the condition for the pyrometer window could be quite different, so that the temperatures in these two works are not directly comparable.

After growth, samples were quenched to room temperature, and transferred under vacuum to the analysis chamber for scanning tunneling microscopy (STM) and Auger spectroscopy study. Auger spectroscopy was measured with a Perkin-Elmer 15-255G system. STM measurements were performed as previously described [2]. X-ray diffraction (XRD) observations are performed on a Philips Materials Research Diffractometer, with Xray source using a Bartels monochromator in four crystal mode.

\section{Results and Discussion}

\subsection{Surface Structures}

Figure 2 shows an STM image of the $\operatorname{InGaN}(0001)$ surface, illustrating two types of surface structures. On the left side of the image there appears a structure with small pits (vacancy islands) and bright double rows, identical to that described in our previous work [4] [7]. The top atomic layer of this structure consists entirely of In atoms, and partial occupation of In in the second layer also occurs. The vacancy islands and double rows arise as a form of strain relief for the second layer In atoms.

On the right side of Figure 2 is a new structure which has not been previously discussed. This structure is observed on surfaces of InGaN films which have been grown with relatively high In-flux. In this structure we do notobserve the vacancy islands or double rows (except for the arrows in Figure 2, discussed below). Rather, the surface is relatively uniform, with $1 \times 1$ symmetry, and displays a number of raised features as shown in Figure 3. The raised features are triangular, with their orientation reversing as one moves across a bilayer step of this high In-content structure.

An important characteristic of this high In-content structure is its height, measured in the STM constantcurrent images, relative to the vacancy island type structure. For the terraces in Figure 1 we find a height difference of $3.5 \AA$, i.e. $0.9 \AA$ larger than an expected bilayer step of $2.6 \AA$. This height difference is, however, found to be voltage dependent; for relatively low bias voltages such as in Figure 1 we observe a height difference of $3.3-3.5 \AA$ whereas for larger bias voltage of $2-3 \mathrm{~V}$ (empty or filled states) we find a height difference of 2.8-2.9 A. Based on these structural characteristics, we propose that the high In-content structure consists of two adlayers on indium (i.e. one more adlayer than shown in Figure 1(b)), together with sparse occupation of In in the third atomic layer. The top In adlayer would be out of registry with the underlying adlayer (i.e. with atoms in the threefold hollow sites of the underlying layer) such that the triangular shaped features seen in Figure 3 are produced from the third layer In atoms. This structure is supported by theoretical calculations described below.

First principles total energy calculations were performed for an In bilayer structure containing $2 \mathrm{ML}$ of In. We refer to this structure as a $\mathrm{T} 1+\mathrm{T} 4$ indium bilayer since the topmost layer has $\mathrm{T} 4$ registry and the second layer has $\mathrm{T} 1$ registry relative to their underlying layers, using the notation of Ref. [3]. The calculated equilibrium height of the T4 layer is $2.68 \AA$ above the $\mathrm{T} 1$ layer, close to that observed experimentally. The slightly larger experimental height is attributed to electronic effects in the tunnel current, indicating that this $\mathrm{T} 1+\mathrm{T} 4$ structure is somewhat more metallic than the vacancy island type structure. In the In-rich limit, where $\mu_{\text {In }}=\mu_{\text {In }}$ (bulk), the calculations indicate that the $\mathrm{T} 1+\mathrm{T} 4$ bilayer is lower in energy than the T1 adlayer structure by 0.16 $\mathrm{eV} / 1 \times 1$ unit cell. Thus, the surface energies of the two structures are equal when $\mu_{\text {In }}=\mu_{\text {In (bulk) }}-0.16 \mathrm{eV}$. Put another way, the $\mathrm{T} 1+\mathrm{T} 4$ bilayer structure is stable with respect to agglomeration into bulk In droplets residing on the T1 adlayer structure. This result is in agreement with the image shown in Figure 2. This conclusion for the In adlayer energetics is somewhat analogous to a result obtained earlier for $\mathrm{Ga}$ adlayers on the $\mathrm{GaN}(0001)$ surface. In that case, the laterally contracted Ga bilayer structure was found to be equal in energy to the laterally contracted Ga monolayer structure for $\mu_{\mathrm{Ga}}=\mu_{\mathrm{Ga} \text { (bulk) }}$ $0.17 \mathrm{eV}[8]$.

A final feature of Figure 2 which we comment upon are the surface depressions indicated by the arrows. The large pit indicated by the black arrow is not so typical of the surface, and we believe it is associated with a defect of some sort. We occasionally see such pits on both the high In-content structure and the vacancy island structure; they have diameter and depth significantly larger than the typical pits which occur on the vacancy island type structure and their origin is unknown. The smaller depressions indicated by white arrows in Figure 2 are seen consistently on the high In-content regions of the surface. Their depth is about $0.6 \AA$, and their diameter of about $3 \mathrm{~nm}$ is comparable to the regular pits which occur on the vacancy island portion of the surface. We speculate that these depressions may arise from an original vacancy island which was not completely filled in during the transition to high In-content structure. In other words, this depression may contain $\mathrm{N}$-vacancies in the fourth atomic layer. While it is likely that these N- 
vacancies are filled in during a later stage of the growth, it is possible that some part of the associated In compositional variation will remain. This situation is expected to have significant consequences on the optical properties of InGaN [7].

Based on XRD measurements, we find a bulk indium concentration of about $10 \%$ for the film pictured in Figure 2 and Figure 3. Since there are two monolayers of In on the surface, it is clear that strong surface segregation of the In occurs. A similar situation occurs for the other InGaN(0001) films we have studied, which contained one monolayer of In on the surface and 0-5\% In in the bulk [4] [7]. The surface segregation arises from the fact that the InN bond is much weaker than the $\mathrm{GaN}$ bond (1.93 eV for $\mathrm{InN}$ and $2.24 \mathrm{eV}$ for $\mathrm{GaN}$ [9]) so that it is energetically favorable for $\mathrm{Ga}$ atoms in the surface layer to exchange with underlying In atoms. Strain considerations also favor the placement of In atoms in the top surface layer [4].

Indium surface segregation also occurs on InGaN $(000 \overline{1})$ films, as has been reported in our earlier work [2]. Additional STM results for that surface are shown in Figure 4. The film shown there was grown at $660^{\circ} \mathrm{C}$, with an $\mathrm{In} /(\mathrm{In}+\mathrm{Ga})$ flux ratio of about $36 \%$. Auger spectroscopy measurements indicate close to 1 monolayer of In atoms on the surface of this film, whereas XRD measurements indicate only about 5\% indium incorporation in the bulk. Again, strong surface segregation of the indium is evident. Note however that the image of Figure 4 displays several different types of atomic features on the surface, labeled A-E. These various features are interpreted using first-principles calculations of surface structures [2] [3] [4] [7]. Atoms of type A are, we believe, the only Ga atoms on the surface. All the rest of the atoms, except possibly those of type $\mathrm{E}$, are indium atoms. A typical surface indium atom, type $\mathrm{B}$, is higher than the $\mathrm{Ga}$ atoms by about $0.10-0.15$ $\AA$, close to the $\sim 0.2 \AA$ increase in height computed for substitution of In for $\mathrm{Ga}$ in the surface layer [2]. The type $\mathrm{C}$ indium atoms in 4 appear brighter than those of type $B$, which we attribute to the presence of subsurface metal atoms. In the metal layer below the surface there are both indium and gallium atoms, and the indium atoms there will push up the surface indium atoms above them and make them look brighter in the STM images. For example, some of the triangular arrangements of type $\mathrm{C}$ atoms may be explained by the presence of an indium atom incorporated just below the topmost $\mathrm{N}$ layer. The type $\mathrm{D}$ indium atoms (surrounding the surface Ga atoms) are higher in the STM topograph by about $0.05 \AA$ than the type B indium atoms. This height difference is consistent with theoretical results indicating a $0.04 \AA$ height difference between indium atoms in a $2 \times 2$ mixed adlayer $(0.5 \mathrm{ML} \mathrm{In}+0.5 \mathrm{ML} \mathrm{Ga})$ compared to indium atoms in a purely indium adlayer [10]. Finally, the type E atoms visible on the surface are adsorbates on top of the surface adlayer, arising from additional metal atoms or possibly from unintentional contaminant atoms.

We observe relatively few of the type E adsorbates, which, if they arise from indium atoms, is consistent with the theoretical expectation that such In adatoms are only weakly bound to the surface. First principles total energy calculations were performed for structures containing 1/4 ML In adatoms above the $1 \times 1$ In adlayer surface. These calculations were performed using a $2 \times 2$ unit cell and indicate that the formation energy of such an adatom is $1.6 \mathrm{eV}$ in the In-rich limit, i.e. for the case of $\mu_{\text {In }}=\mu_{\text {In (bulk). In other words, the energy cost to move }}$ an In atom from a bulk In reservoir (such as an In droplet) to an adatom site on a terrace is approximately 1.6 $\mathrm{eV}$. Since the cohesive energy of bulk In is $2.5 \mathrm{eV}$, the binding energy of such an In adatom is therefore quite small, $0.9 \mathrm{eV}$. Such In adatoms will thus either agglomerate into droplets or evaporate. We therefore expect the In adatom-on-adlayer density to be extremely low, in agreement with the experimental results. This situation is in contrast to the clean $\operatorname{GaN}(000 \overline{1})$ surface, where a variety of $\mathrm{Ga}$ adatom-on-adlayer structures, e.g. the $3 \times 3$ structure, are possible [11]. This difference in behavior is attributable to the larger size of the In atoms compared to $\mathrm{Ga}$, since in the $\mathrm{GaN}(000 \overline{1}) 3 \times 3$ structure the $\mathrm{Ga}$ adatoms enable substantial lateral relaxation of the $\mathrm{Ga}$ atoms in the adlayer - an energy lowering mechanism not available for the case of In adatoms on the In adlayer. The absence of In adatoms on the In adlayer of the InGaN(000 $\overline{1})$ surface is also in contrast to the results above for the $\mathrm{InGaN}(0001)$ surface, in which either 1 or 2 indium adlayers are found to be stable. That difference arises from the different bonding character of the first In adlayer in the two cases.

\subsection{Incorporation Kinetics}

Determination of the dependence of indium incorporation on growth parameters is important for controllably growing InGaN with desired indium concentration. It also provides valuable information on growth kinetics, as will be shown below. Similar to what we have done for $(000 \overline{1})$ polar films in our previous work [2], several series of samples were grown here with different growth parameters for (0001) polar films. After the growth, XRD was performed to measure the indium incorporation. For the analysis of the XRD data, we assume that InGaN layer is fully strain relaxed (the thickness of InGaN is typically $100 \mathrm{~nm}$ ), and that Vegard's law applies.

In this study two growth parameters were varied, the substrate temperature and group III/V ratio. The $\mathrm{N}_{2}$ flux 
is kept constant to maintain the growth chamber pressure of $1.8 \times 10^{-5}$ Torr, and the $\mathrm{In} /(\mathrm{In}+\mathrm{Ga})$ flux ratio is kept at 33\% when In and Ga fluxes are both varied. The results are shown in Figure 5(a). At a given In and Ga flux, when the substrate temperature is increased, the indium incorporation decreases. More interestingly, when temperature is kept constant, and both In and Ga fluxes are increased while keeping their ratio constant, it is found the indium incorporation increases at low metal flux, but decreases at high metal flux. It is well known that $\mathrm{GaN}$ growth goes through a smooth/rough transition when $\mathrm{Ga} / \mathrm{N}$ ratio is unity [12], so by measuring the $\mathrm{Ga}$ flux at the transition point, the active $\mathrm{N}$ flux can be determined. An active $\mathrm{N}$ flux of $2.6 \times 10^{14} \mathrm{~cm}^{-2} \mathrm{~s}^{-1}$ is thus found experimentally (with an uncertainty of about $10 \%)$. The results of Figure 5(a) are similar to what we observed for (0001) polar films [2] - indium incorporation increases when the total metal flux is increased under $\mathrm{N}$ rich conditions, but decreases under metal rich conditions - as pictured in Figure 6(a). Figures 5(b) and 6(b) shows results from a theoretical model, described below.

Figure 7 illustrates the near surface region of an $\operatorname{InGaN}(0001)$ film, showing a typical distribution of metal atoms (indium and gallium) on the surface and in the bulk. We assume for ease of illustration the situation with a single In adlayer, although the results are the same if we have two In adlayers (i.e. the high In-content structure). Our surface is then terminated with two monolayers of metal atoms (layer 2 and 3 in Figure 7), and during growth a few additional metal atoms (layer 1) may reside on top of this monolayer. The population of metal atoms in layer 1 depends on how metal rich the growth is. For the present case of InGaN growth, the observed surface segregation reveals that layer 2 (and layer 1) contain mainly In atoms.

In general, the In composition $x$ in the alloy is given by a ratio of incorporation rates for In atoms to the total incorporation rate for metal (i.e. $\mathrm{In}+\mathrm{Ga}$ ) atoms. The former we write as $f_{\mathrm{In}}-R_{\mathrm{In}}$, where $f_{\mathrm{In}}$ is the incident flux of In and $R_{\text {In }}$ is the rate of In loss from the film. Loss may occur through either evaporation or droplet formation, and one can further distinguish losses from the different surface layers of the film (i.e. layers 1 or 2 for In, as shown in Figure 7). The total metal incorporation rate is similarly written as $f_{\mathrm{In}}-R_{\mathrm{In}}+f_{\mathrm{Ga}}-R_{\mathrm{Ga}}$ where $f_{\mathrm{Ga}}$ is the incident flux of Ga atoms and $R_{\mathrm{Ga}}$ is the loss rate for $\mathrm{Ga}$. For growth of a stoichiometric film, we have

$$
f_{\mathrm{In}}-R_{\mathrm{In}}+f_{\mathrm{Ga}}-R_{\mathrm{Ga}}=f_{\mathrm{N}}-R_{\mathrm{N}}
$$

where $f_{\mathrm{N}}$ is the incident flux of active $\mathrm{N}$ and $R_{\mathrm{N}}$ is the loss rate of $\mathrm{N}$ atoms. Thus for the indium composition we have in general

$$
x=\frac{f_{\mathrm{In}}-R_{\mathrm{In}}}{f_{\mathrm{In}}-R_{\mathrm{In}}+f_{\mathrm{Ga}}-R_{\mathrm{Ga}}}=\frac{f_{\mathrm{In}}-R_{\mathrm{In}}}{f_{\mathrm{N}}-R_{\mathrm{N}}} .
$$

Since there is a strong surface segregation of indium atoms, the gallium atom population on the surface is small, under the condition that the growth is not overly metal rich (we consider in this work cases where $f_{\mathrm{Ga}}<f$ $\left.{ }_{N}\right)$. Then, the gallium evaporation will also be small, and most of the incident gallium flux will incorporate into the bulk. We thus take $R_{\mathrm{Ga}}=0$ for both the metal rich and nitrogen rich situations discussed below.

Let us consider indium incorporation in the metal rich regime. In that case, the maximum amount of In+Ga which can be incorporated into the bulk is limited by the active nitrogen flux. Since the surface is metal rich, we expect minimal loss of $\mathrm{N}$ atoms, so that $R_{\mathrm{N}}=0$. Thus, the rate of metal incorporation is simply equal to the flux of active nitrogen, $f_{\mathrm{N}}$. When both indium and gallium fluxes are increased, those additional gallium atoms will compete to go into bulk. Since there is strong indium surface segregation, those additional gallium atoms will mostly go into the bulk and kick out indium atoms, so that the indium incorporation will decrease. From Eq. (1) we have for the rate of In incorporation $f_{\mathrm{In}}-R_{\mathrm{In}}=f_{\mathrm{N}}-f_{\mathrm{Ga}}$. Therefore, the indium concentration incorporated into bulk is given by

$$
x=\frac{f_{\mathrm{N}}-f_{\mathrm{Ga}}}{f_{\mathrm{N}}}
$$

$$
\left(f_{\mathrm{Ga}}+f_{\mathrm{In}}-R_{\mathrm{In}}>f_{\mathrm{N}}\right)
$$

Excess indium atoms, formed from the increased metal flux as well as the decreased bulk incorporation, will tend to increase the concentration of In atoms in layer 1 , and so loss from that layer will also increase. The excess indium will evaporate, or alternatively, it will form droplets if there is too much indium. This result is consistent with that of Böttcher et al.'s [13], although the latter didn't distinguish the case of metal rich and $\mathrm{N}$ rich conditions.

In the nitrogen rich region, the number of metal atoms in layer 1 is minimal. Thus, metal evaporation mainly proceeds via layer 2 , for which the loss rate is 
constant for a given temperature since the number of In atoms in layers 2 is constant (close to 1 monolayer) [a]

The rate of In incorporation is thus given by $f_{\mathrm{In}}-R_{\text {In }}$ , and the rate of total metal incorporation is given by $f_{\mathrm{Ga}}$ $+f_{\text {In }}-R_{\text {In }}$. Therefore, the indium concentration in the bulk can be described by

$$
x=\frac{f_{\mathrm{In}}-R_{\mathrm{In}}}{f_{\mathrm{Ga}}+f_{\mathrm{In}}-R_{\mathrm{In}}} \quad\left(f_{\mathrm{Ga}}+f_{\mathrm{In}}-R_{\mathrm{In}}<f_{\mathrm{N}}\right)
$$

where $R_{\text {In }}$ is treated as a parameter. With the loss of indium being constant, an increase of both indium and gallium flux while keeping their ratio constant will thus lead to an increase in the indium incorporation.

Theoretical curves based on equation (3) and equation (4) are shown in Figure 5(b). Parameters used are: $f$ $\mathrm{N}=2.6 \times 10^{14} \mathrm{~cm}^{-2} \mathrm{~s}^{-1} ; R_{\mathrm{In}}=0.6 \times 10^{14} \mathrm{~cm}^{-2} \mathrm{~s}^{-1}$ for $\mathrm{T}=$ $620^{\circ} \mathrm{C} ; R_{\text {In }}=0.3 \times 10^{14} \mathrm{~cm}^{-2} \mathrm{~s}^{-1}$ for $\mathrm{T}=600^{\circ} \mathrm{C} ; R_{\text {In }}=$ $0.04 \times 10^{14} \mathrm{~cm}^{-2} \mathrm{~s}^{-1}$ for $\mathrm{T}=580^{\circ} \mathrm{C}$. All these parameters were adjusted to get a close fit to the experimental results. It should be pointed out that the active $\mathrm{N}$ flux obtained from the incorporation curve fitting is the same as that determined from smooth/rough transition experiment described above. The deduced variation of $R_{\text {In }}$ with growth temperature is consistent with a thermal activation barrier of about $4 \mathrm{eV}$, close to the result of Averbeck et al. of 3.5-3.8 eV [14]. Comparing Figure 5(a) and (b), we find the theoretical curves give a good fit to the experimental results.

The model described here can also be used to fit the $(000 \overline{1})$ polarity data shown in Figure 6(a), with the theoretical results displayed in Figure 6(b). Parameters used are: $f_{\mathrm{N}}=2.9 \times 10^{14} \mathrm{~cm}^{-2} \mathrm{~s}^{-1} ; R_{\mathrm{In}}=1.0 \times 10^{14} \mathrm{~cm}^{-2} \mathrm{~s}^{-1}$ for $\mathrm{T}=660^{\circ} \mathrm{C} ; R_{\text {In }}=0.5 \times 10^{14} \mathrm{~cm}^{-2} \mathrm{~s}^{-1}$ for $\mathrm{T}=640^{\circ} \mathrm{C} ; R_{\mathrm{In}}$ $=0$ for $\mathrm{T}=620^{\circ} \mathrm{C}$. Again, the $R_{\text {In }}$ decrease with temperature was chosen to fit the experimental data. Comparing Figure 6(a) and (b), we see the overall trend that the indium incorporation first increases then decreases with increasing metal flux is seen in both experiment and theory. However, we point out that for temperatures of 620 and $660^{\circ} \mathrm{C}$ there is a discrepancy between the experimental results and theory in that the decrease of the incorporation at low fluxes, as a function of decreasing metal flux, is too fast and slow, respectively, compared to the theory. The reason for this discrepancy is not understood at present, although it may arise from some limitation in the kinetic model.

\section{Conclusion}

InGaN (0001) and (000) $)$ surfaces are studied by STM. We find that the surfaces are mostly covered by indium atoms, thus revealing strong surface segregation of the indium. For In-GaN(0001) surfaces prepared under Inrich conditions a new surface structure is observed, and based on experimental and theoretical results it is identified as containing two adlayers of In. The dependence of the indium incorporation on growth temperature and group III/V ratio is reported, and explained by a quantitative model based on the observed surface segregation.

\section{ACKNOWLEDGMENTS}

This work was supported by the National Science Foundation (grant DMR-9985898), the Office of Naval Research (grant N00014-96-1-0214, monitored by Colin Wood), and the German Science Foundation (focused project "Group-III Nitrides").

\section{REFERENCES}

[a] The argument of the surface always being terminated by at least two layers of metal atoms may not be true under very $\mathrm{N}$ rich conditions, since in that case we see a different surface reconstruction $3 \times 3$ which may not contain the In adlayer, as discussed in Ref. [4].

[1] Shuji Nakamura, Gerhard Fasol, The Blue Laser Diode - GaN based Light Emitters and Lasers , (Springer-Verlag, Heidelberg, 1997), .

[2] Huajie Chen, A.R. Smith, R.M. Feenstra, D.W. Greve, J.E. Northrup, MRS Internet J. Nitride Semicond. Res. 4S1, G9.5 (1999).

[3] JE Northrup, J Neugebauer, Phys. Rev. B 60, 8473-8476 (1999).

[4] H Chen, RM Feenstra, JE Northrup, T Zywietz, J Neugebauer, DW Greve, J. Vac. Sci. Technol. B 18, 2284-2289 (2000).

[5] V Ramachandran, MF Brady, AR Smith, RM Feenstra, DW Greve, J. Electron. Mater. 27, 308-312 (1998).

[6] V Ramachandran, AR Smith, RM Feenstra, J. Vac. Sci. Technol. A 17, 1289-1293 (1999).

[7] H Chen, RM Feenstra, JE Northrup, T Zywietz, J Neugebauer, Phys. Rev. Lett. 85, 1902-1905 (2000).

[8] JE Northrup, J Neugebauer, RM Feenstra, AR Smith, Phys. Rev. B 61, $9932-9935$ (2000).

[9] W.A. Harrison, Electronic Structure and the Properties of Solids, (W. H. Freeman and Company, San Francisco, 1980), .

[10] T. Zywietz, Ph.D. Thesis, Technical University of Berlin, 1999

[11] A. R. Smith, R. M. Feenstra, D. W. Greve, J. Neugebauer, J. E. Northrup, Phys. Rev. Lett. 79, 3934 (1997).

[12] EJ Tarsa, B Heying, XH Wu, P Fini, SP DenBaars, JS Speck, J. Appl. Phys. 82, 5472-5479 (1997).

[13] T Boettcher, S Einfeldt, V Kirchner, S Figge, H Heinke, D Hommel, H Selke, PL Ryder, Appl. Phys. Lett. 73, 3232-3234 (1998). 
[14] R. Averback, H. Riechert, Phys. Stat. Sol. A 176, 301 (1999).

\section{FIGURES}

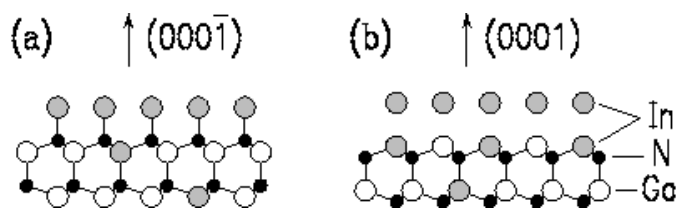

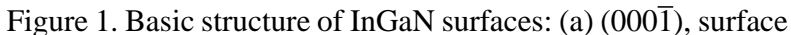
is terminated by one monolayer of indium atoms. (b) (0001), surface is terminated with two layers of metal atoms, with the top layer being entirely In, and second layer containing a mixture of In and Ga.

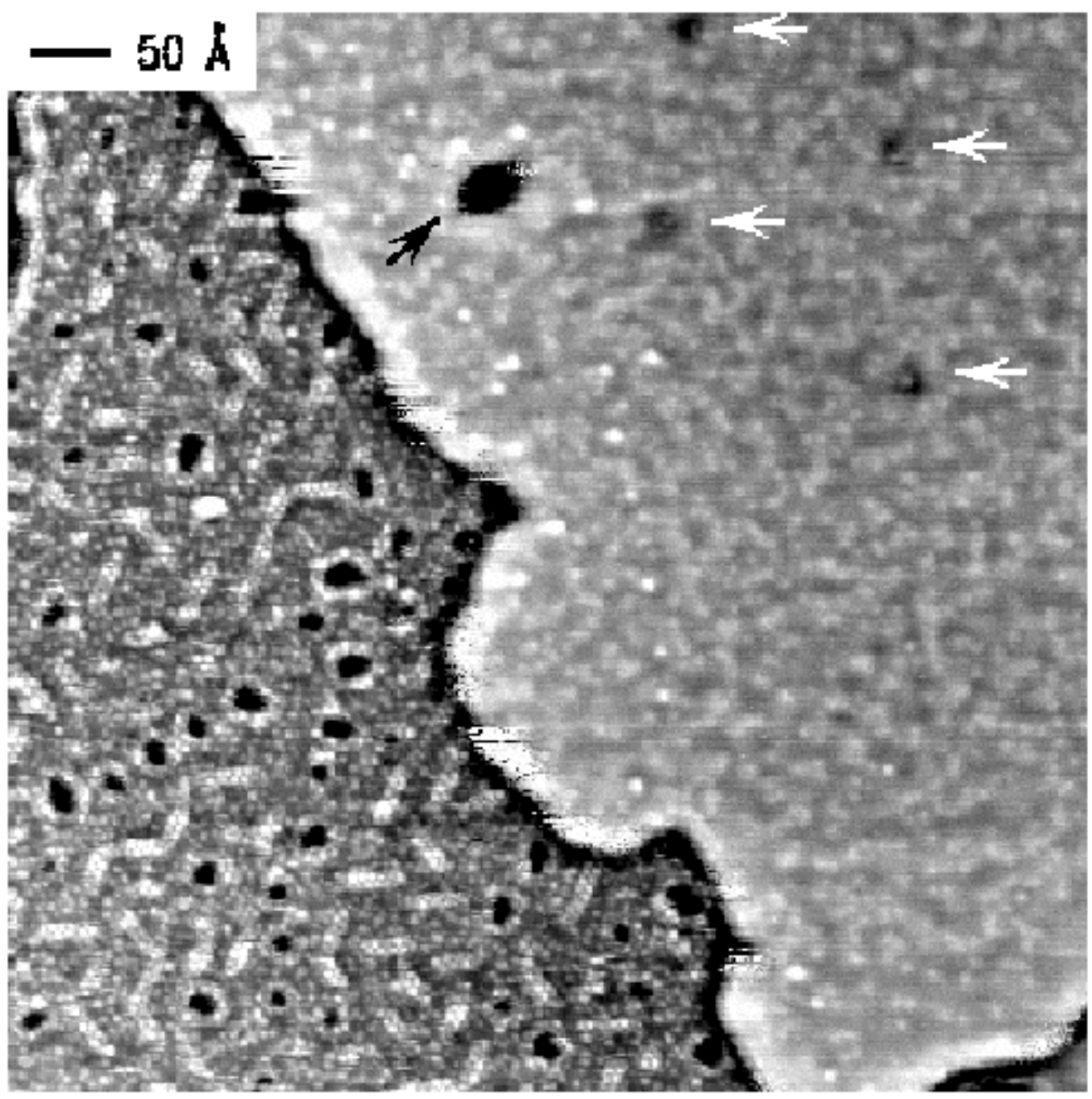

Figure 2. STM image of an $\mathrm{InGaN}(0001)$ surface with relatively high In-content, acquired with sample bias voltage of $+0.8 \mathrm{~V}$ and tunneling current of $0.075 \mathrm{nA}$. Some local background subtraction has been applied to the image, to permit viewing of the features on both terraces. The grey scale range on a given terrace is $1.1 \AA$. Sample was grown at $600^{\circ} \mathrm{C}$ with Ga and In fluxes of $8.3 \times 10^{13}$ and $1.3 \times 10^{14} \mathrm{~cm}^{-2} \mathrm{~s}^{-1}$ respectively. 


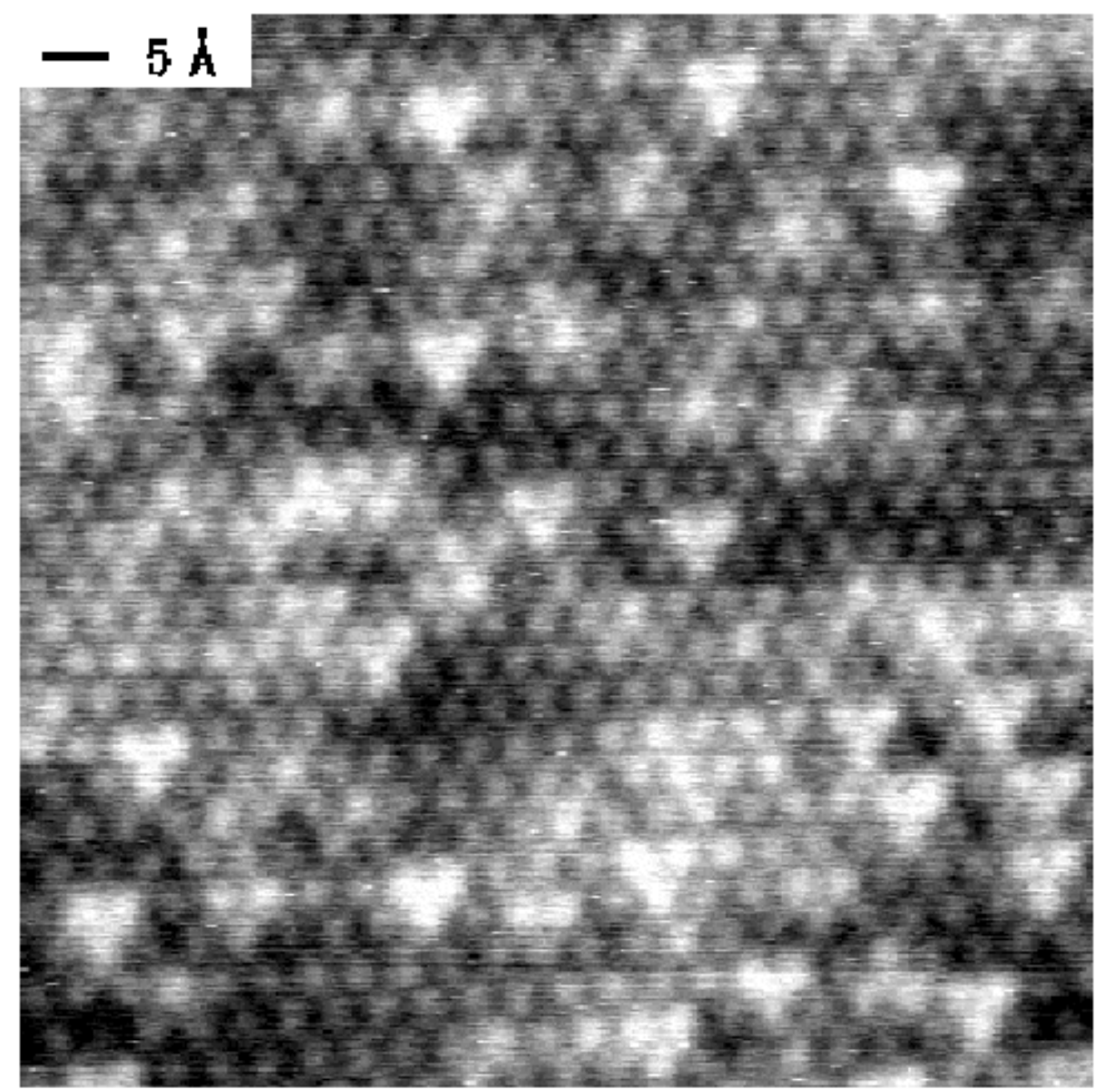

Figure 3. STM image of InGaN(0001) surface, from the same film as pictured in Figure 2. Image acquired with sample bias voltage of $-0.1 \mathrm{~V}$ and tunneling current of $0.075 \mathrm{nA}$. The grey scale range is $0.35 \AA$. 


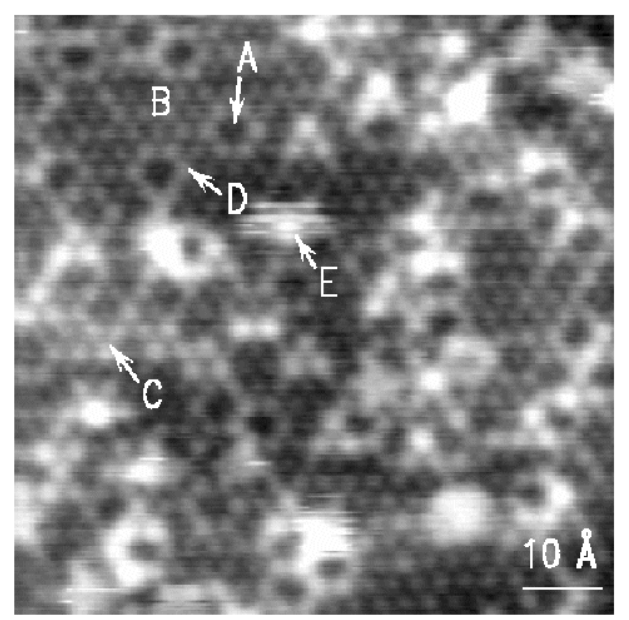

Figure 4. STM image of $\operatorname{InGaN}(000 \overline{1})$ surface from a sample with $5 \%$ indium incorporation in the bulk. Sample bias voltage is $-1.0 \mathrm{~V}$ and tunneling current is $0.15 \mathrm{nA}$. The grey scale range is $0.6 \AA$. Symbols A-E denote different types of atoms, as described in the text.
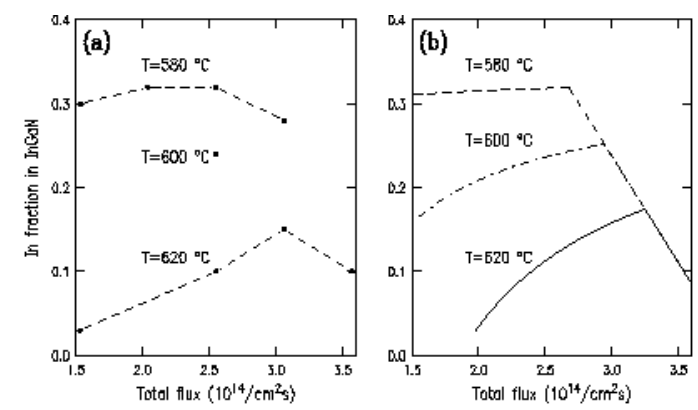

Figure 5. (a) Indium incorporation dependence on ( $\mathrm{In}+\mathrm{Ga})$ flux for (0001) polarity $\mathrm{InGaN}$. The $\mathrm{In} /(\mathrm{In}+\mathrm{Ga})$ flux ratio was kept constant at $33 \%$. (b) Theoretical curves based on Eqs. (3) and (4) in the text.
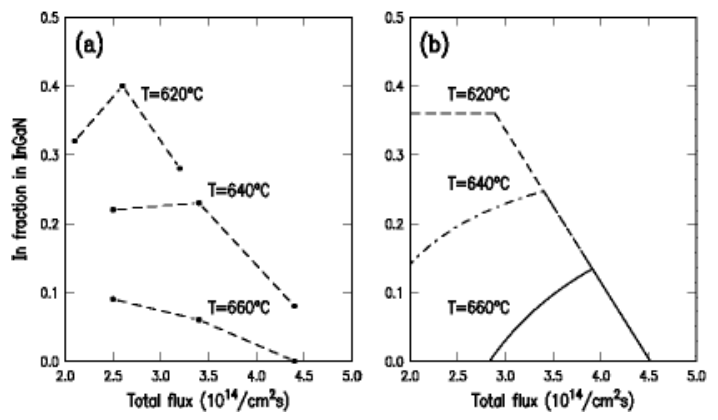

Figure 6. (a) Indium incorporation dependence on (In+Ga) flux for $(000 \overline{1})$ polarity $\mathrm{InGaN}$. The $\mathrm{In} /(\mathrm{In}+\mathrm{Ga})$ flux ratio was kept constant at $36 \%$. (b) Theoretical curves based on Eqs. (3) and (4) in the text.

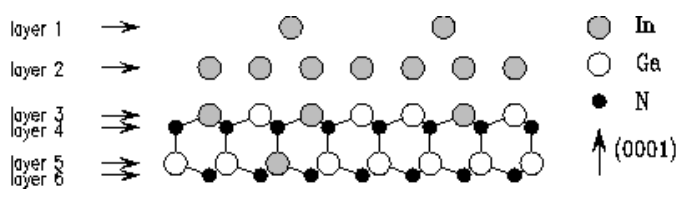

Figure 7. Schematic view of the $\operatorname{InGaN}(0001)$ surface layers: layer 1 , indium adatoms residing on top of the indium adlayer; layer 2, indium adlayer; layer 3, metal (In or $\mathrm{Ga}$ ) atoms; layers 4 and 6 , nitrogen atoms; layer 5 , metal atoms. 